\title{
Geometrization of gravito-electromagnetic interactions from boundary conditions in the variational principle
}

\author{
Jesús Martín Romero ${ }^{2, a}$, Luis Santiago Ridao ${ }^{2, b}$, Mauricio Bellini ${ }^{1,2, c}$ \\ ${ }^{1}$ Departamento de Física, Facultad de Ciencias Exactas y Naturales, Universidad Nacional de Mar del Plata, Funes 3350 (7600), Mar del Plata, \\ Argentina \\ ${ }^{2}$ Instituto de Investigaciones Físicas de Mar del Plata (IFIMAR), Consejo Nacional de Investigaciones Científicas y Técnicas (CONICET), Buenos \\ Aires, Argentina
}

Received: 5 June 2019 / Accepted: 21 July 2019 / Published online: 7 August 2019

(C) The Author(s) 2019

\begin{abstract}
We study the conditions of integrability when the boundary terms are considered in the variation of the geometric contribution of the Einstein-Hilbert action. We explore the emergent physical dynamics that is obtained when we make a displacement from a background Riemann manifold to an extended one, on which the non-metricity is nonzero. Under these circumstances, a classical description of the electrodynamics and non-perturbative gravitational waves is considered in the extended manifold, when we vary the action.
\end{abstract}

\section{Introduction and motivation}

Geometrodynamics [1,2] is a picture of general relativity that studies the evolution of the spacetime geometry. The key notion of geometrodynamics was the idea of charge without charge, in which the Maxwell field was taken to be free of sources, and hence a non-vanishing charge could only arise from an electric flux line trapped in the topology of spacetime. With the construction of ungauged supergravity theories, it was realized that the Abelian gauge fields in such theories were source-free, and so the charges arising therein were therefore central charges [3]. The significant advantages of geometrodynamics usually come at the expense of manifest local Lorentz symmetry [4]. During the 1970s and 1980s a method of quantization was developed in order to deal with some unresolved problems of quantum field theory in curved spacetimes [5-7]. Quantum geometrodynamics, introduced by Wheeler $[2,8]$, and quantum geometry $[9,10]$ are some of the geometrical frameworks involved. Recently, a quantum unified spinor field (USF) was devel-

\footnotetext{
a e-mail: jesusromero@ conicet.gov.ar

b e-mail: santiagoridao@hotmail.com

c e-mail: mbellini@mdp.edu.ar
}

oped (see [11] and the references therein), where a quantum spinor field with components $\hat{\Psi}_{\alpha}$ is responsible for the displacement from the Riemannian manifold to an extended one. These fields are defined on the background spacetime, emerging from the expectation value of the quantum structure of spacetime generated by matrices that comply with a Clifford algebra. In this framework, it was demonstrated that spinor fields are candidate to describe all known interactions in physics, gravitation included, and a non-linear description of gravitational waves can be done. However, in this work we shall refer only to classical physics. The boundary conditions in the minimum action principle are a very important issue which must be taken into account to develop a physical theory $[12,13]$. In this framework, the conditions of the integrability of the boundary conditions are relevant. In this paper we aim to study the possible electromagnetic physics which can be obtained when we consider a displacement from a background Riemannian manifold, such that the non-metricity on the extended manifold is nonzero.

The work is organized as follows: In Sect. 2 we study the geometrical origin of sources in the geodesic equations which can be related to nontrivial boundary conditions in the minimal action principle. In Sect. 3, we show that the Einstein equations with cosmological parameter included in the absence of physical sources on a Riemannian manifold are equivalent to these equations with sources, but with a different cosmological parameter, when the extended manifold is displaced with respect to the Riemann one by contortional terms. In Sect. 4, we study massless gravitational waves equations in this context. In Sect. 5 we study a particular case where the contortion term, which characterizes the extended manifold, originates with electromagnetic fields. Finally, in Sect. 6 we present some final remarks. 


\section{Boundary conditions in the geometrical action}

In a previous paper we have studied the dynamics obtained from a variational principle over the Einstein-Hilbert (EH) action, where non-metricity [16] is considered. In this work, we shall deal with an extended geometry, by considering only the geometric part of the EH action,

$\mathcal{S}=\frac{1}{2 \kappa} \int_{V} \mathrm{~d}^{4} x \sqrt{-g} \mathcal{R}$,

in which $\mathcal{R}$ is the scalar curvature of the complete (nonRiemannian) connection. The variational principle is expressed as $\delta \mathcal{S}=0$, with

$$
\begin{aligned}
\delta \mathcal{S}= & \frac{1}{2 \kappa} \int_{V} \mathrm{~d}^{4} x \sqrt{-g}\left(\delta g^{\alpha \beta} G_{\alpha \beta}\right. \\
& \left.+g^{\alpha \beta} \delta \bar{R}_{\alpha \beta}+g^{\alpha \beta} \delta \Delta R_{\alpha \beta}\right),
\end{aligned}
$$

in which

$R_{\alpha \beta}=\bar{R}_{\alpha \beta}+\Delta R_{\alpha \beta}$

$\overline{\mathcal{R}}$ being the Riemannian scalar curvature and $\Delta \mathcal{R}$ being the part of $\mathcal{R}$ which is exclusively non-Riemannian. The interesting point is that new physics is obtained from the last two terms of the r.h.s. of Eq. (2). We shall refer to this issue below.

Now we consider Eq. (2) in the presence of non-metricity. The variation of the action (1) is

$\delta \mathcal{S}=\int_{V} \mathrm{~d}^{4} x \sqrt{-g} G_{\alpha \beta} \delta g^{\alpha \beta}+\int_{V} \mathrm{~d}^{4} x \sqrt{-g} N_{\mu \alpha \beta} W^{\beta \mu \alpha}$,

in which the auxiliary tensor $W_{\alpha \beta}^{\mu}$ is defined through the variation of the connections $\Gamma_{\alpha \beta}^{\mu}$ by

$W_{\alpha \beta}^{\mu}=\delta \Gamma_{\alpha \beta}^{\mu}-\delta \Gamma_{\sigma \beta}^{\sigma} \delta_{\alpha}^{\mu}$.

An extra term must be introduced in Eq. (4), in order to take into account the torsion effects. However, in this work those contributions will not be considered. Furthermore, we shall neglect the surface term in Eq. (4). This could be interpreted as a source of the cosmological parameter in a gaussian hypersurface. The surface term is obtained in virtue of the Stokes theorem. When the condition of integrability is fulfilled, the boundary terms in (4) take the form

$$
\int_{V} \mathrm{~d}^{4} x \sqrt{-g}\left(W^{\mu}\right)_{\mid \mu}=\int_{\partial V} \mathrm{~d}^{3} x \sqrt{-g} W^{\mu} n_{\mu},
$$

where $n_{\mu}$ is a vector field which is normal to the 3D hypersurface $\partial V$, involving the manifold of the 4D volume $V$, and "|" denotes the covariant derivatives with respect to the Riemann manifold defined by the Levi-Civita connections $\left\{\begin{array}{c}\mu \\ \alpha \beta\end{array}\right\}$ and $W^{\alpha}=\delta \hat{\Gamma}_{\beta \epsilon}^{\epsilon} g^{\beta \alpha}-\delta \hat{\Gamma}_{\beta \gamma}^{\alpha} g^{\beta \gamma}$. It is quite usual in the literature, in the framework of the two cosmological astrophysical scenarios, to assume that the surface integral must be neglected because the distance from any matter to $\partial V$ can be considered large enough to say that $W^{\mu} \rightarrow 0$ in such a limit. However, the 3D hypersurface term could be a source for the cosmological parameter $[17,18]$.

To describe the idea in terms of the geodesic dynamics, we can consider the geodesic equations in the presence of an external force on a Riemann manifold. The equations take the form

$\frac{\partial^{2} x^{\mu}}{\partial S^{2}}+\left\{\begin{array}{c}\mu \beta \\ \alpha \beta\end{array}\right\} U^{\alpha} U^{\beta}=f^{\mu}$,

in which $f^{\mu}$ are the components of the external force, which we consider as related with some interaction. In this work we shall deal only with electromagnetic interactions. The geodesics of Eq. (7) give us the path of a test particle over a gravitational background and describes the influence of an external and non-gravitational force.

Our purpose is to extend the theory in order to obtain a more general geometrical frame in an extended manifold where the new connection dynamics would be absent if the external force is. The new extended geodesic is

$\frac{\partial^{2} x^{\mu}}{\partial S^{2}}+\Gamma_{\alpha \beta}^{\mu} U^{\alpha} U^{\beta}=0$,

in which $\Gamma_{\alpha \beta}^{\mu}$ is a non-Riemannian connection. The Riemannian and non-Riemannian connections are related through

$\Gamma_{\alpha \beta}^{\mu}=\left\{\begin{array}{l}\mu \beta \\ \alpha \beta\end{array}+K_{\alpha \beta}^{\mu}\right.$,

where $K_{\alpha \beta}^{\mu} \equiv \delta \Gamma_{\alpha \beta}^{\mu}$ are the components of the contortion tensor, which are responsible for the displacement from the Riemann manifold [defined by the Levi-Civita connections $\left\{\left\{_{\alpha \beta}^{\mu}\right\}\right]$, to the extended manifold defined by the connections $\Gamma_{\alpha \beta}^{\mu}$. Notice that $\delta \Gamma_{\alpha \beta}^{\mu}$ are not variations in the sense of a series development, so that our formalism will be non-perturbative. In general, the contortion contributions originate with the torsion and non-metricity, which are defined by $[14,15]$

$$
\begin{aligned}
K_{\alpha \beta}^{\mu}= & -\frac{g^{\nu \mu}}{2}\left\{T_{\beta \nu}^{\rho} g_{\alpha \rho}\right. \\
& \left.+T_{\alpha \nu}^{\rho} g_{\rho \beta}-T_{\beta \alpha}^{\rho} g_{\rho \nu}+N_{\alpha \nu \beta}+N_{\nu \beta \alpha}-N_{\alpha \beta \nu}\right\},
\end{aligned}
$$

with

$$
\begin{aligned}
T_{\alpha \beta}^{\mu} & =\Gamma_{\beta \alpha}^{\mu}-\Gamma_{\alpha \beta}^{\mu}, \\
N_{\alpha \beta \gamma} & =g_{\beta \gamma ; \alpha},
\end{aligned}
$$

for a coordinate basis. In order to obtain a path which is coherent with the background source and the interaction, we must choose non-Riemannian connections according to

$K_{\alpha \beta}^{\mu} U^{\alpha} U^{\beta}=-f^{\mu}$.

We could suppose that we are dealing with observers such that $|U|^{2}=1$, so that

$K_{\alpha \beta}^{\mu}=-f^{\mu} U_{\alpha} U_{\beta}$. 
We must remark that Eq. (14) is not the more general prescription, but the simplest one in which the new connection is torsion-free, and all the non-Riemannian contributions are related to the non-metricity. The non-metricity takes the form

$$
\begin{aligned}
g_{v \pi ; \rho} & =g_{v \pi \mid \rho}-K_{v \rho}^{\mu} g_{\mu \pi}-K_{\pi \rho}^{\mu} g_{v \mu} \\
& =\left(f_{\pi} U_{\nu}+f_{\nu} U_{\pi}\right) U_{\rho},
\end{aligned}
$$

where we have used Eq. (14). The presence of non-metricity is very important, because it might be the reason why the theory is not integrable [see Appendix A].

\section{Einstein equations from a non-Riemannian geometry}

In this work we shall study the possible origin of the cosmological parameter due exclusively to the non-metricity of the extended variety. The study of surface terms in the action with the use of the specific geometry that we develop in the present paper could be the object of further work. Under previous considerations we make the action extreme, $\delta S=0$, and we obtain

$R_{\alpha \beta}-\frac{1}{2} \mathcal{R} g_{\alpha \beta}+\Lambda_{1}(x) g_{\alpha \beta}=0$.

Notice that all tensors are defined with respect to the nonRiemannian complete connections. The extended Einstein equations (16) are obtained in the absence of matter in the frame of the non-Riemannian geometry, but they could be re-interpreted as the effective Einstein equations in the Riemannian manifold, in the presence of matter,

$\bar{R}_{\alpha \beta}-\frac{1}{2} \overline{\mathcal{R}} g_{\alpha \beta}+\Lambda_{1}(x) g_{\alpha \beta}=k \bar{T}_{\alpha \beta}$.

Here we must remark that $\bar{T}_{\alpha \beta}$ is geometrically induced by the non-Riemannian characteristics of the manifold which has no geometric explanation in the Riemannian frame: those observers who build physics with the Levy-Civita connections of the Riemannian manifold will interpret $\bar{T}_{\alpha \beta}$ as the source of the physical fields. Therefore $\bar{T}_{\alpha \beta}$ can be viewed as a geometrically induced energy-momentum tensor that is the source of matter. This is due exclusively to the contortion terms, $K_{\alpha \beta}^{\mu}$ :

$k \bar{T}_{\alpha \beta}=-K_{\mu \beta \mid \alpha}^{\mu}+K_{\alpha \beta \mid \mu}^{\mu}-K_{\mu \beta}^{\sigma} K_{\sigma \alpha}^{\mu}+K_{\alpha \beta}^{\sigma} K_{\sigma \mu}^{\mu}$.

The contribution of the cosmological parameter must be taken into account:

$\Lambda_{1}(x) g_{\alpha \beta} \delta g^{\alpha \beta}=N_{\alpha \beta \mu} W^{\mu \alpha \beta}$,

where we can distinguish between Riemannian and nonRiemannian parts of the tensor $W^{\mu \alpha \beta}=\bar{W}^{\mu \alpha \beta}+\Delta W^{\mu \alpha \beta}$. Then it is easy to see that $N_{\alpha \beta \mu} \Delta W^{\mu \alpha \beta}=0$, and the Eq. (19) is reduced to $\Lambda_{1}(x) g_{\alpha \beta} \delta g^{\alpha \beta}=N_{\alpha \beta \mu} \bar{W}^{\mu \alpha \beta}$. On the other hand, we could identify another contribution to the cosmological parameter provided by an extra contribution of the terms that induce the effective energy-momentum tensor:

$$
\begin{aligned}
\Lambda_{2}(x) & =\frac{1}{2} g^{\lambda \gamma}\left(K_{\mu \gamma \mid \lambda}^{\mu}-K_{\lambda \gamma \mid \mu}^{\mu}+K_{\mu \gamma}^{\sigma} K_{\sigma \lambda}^{\mu}-K_{\lambda \gamma}^{\sigma} K_{\sigma \mu}^{\mu}\right) \\
& \equiv-\frac{k}{2} g^{\lambda \gamma} \bar{T}_{\lambda \gamma} .
\end{aligned}
$$

The total cosmological parameter is $\Lambda(x)=\Lambda_{1}(x)+$ $\Lambda_{2}(x)$. We can notice that both cosmological parameters, $\Lambda_{1}$ and $\Lambda_{2}$, originate with the existence of a nonzero nonmetricity. Therefore, we find that the Einstein equations can be re-written with a variable cosmological parameter $\Lambda(x)$ in the absence of physical sources,

$\bar{R}_{\alpha \beta}-\frac{1}{2} \overline{\mathcal{R}} g_{\alpha \beta}+\Lambda(x) g_{\alpha \beta}=0$,

where the $\Lambda_{2}(x)$-term, which originates with the contortion, was absorbed into the cosmological parameter $\Lambda(x)$.

\section{Gravitational waves}

In order to describe gravitational waves [19-26], we shall use the fact that the Riemannian metric tensor is $\bar{g}_{\alpha \beta}=$ $g_{\alpha \beta}+\delta g_{\alpha \beta}$, to write

$0=\bar{g}_{\alpha \beta \mid \gamma}=g_{\alpha \beta \mid \gamma}+\delta g_{\alpha \beta \mid \gamma}$,

where the covariant derivatives are done with respect to the Levi-Civita connections $\left\{\begin{array}{l}\alpha \gamma \\ \beta \gamma\end{array}\right\}=\Gamma_{\beta \gamma}^{\alpha}-K_{\beta \gamma}^{\alpha}$. Therefore, we obtain

$\delta g_{\alpha \beta \mid \gamma}=\frac{1}{2} G_{\alpha \beta \gamma \nu \sigma} \delta g^{\nu \sigma}$

where we have used the fact that

$$
\begin{aligned}
G_{\alpha \beta \gamma \nu \sigma}= & g_{\nu \beta}\left(g_{\alpha \sigma, \gamma}+g_{\gamma \sigma, \alpha}-g_{\beta \gamma, \sigma}\right) \\
& +g_{\nu \alpha}\left(g_{\beta \sigma, \gamma}+g_{\gamma \sigma, \beta}-g_{\beta \gamma, \sigma}\right) .
\end{aligned}
$$

Therefore, we massive equation of motion for gravitational waves is

$\bar{\square} \delta g_{\alpha \beta}+\Xi_{\alpha \beta}^{\mu v} \delta g_{\mu \nu}=0$,

with $\Xi_{\alpha \beta}^{\mu \nu}=\frac{g^{\gamma \rho}}{2}\left(G_{\alpha \beta \gamma}{ }^{\mu \nu}{ }_{\mid \rho}+\frac{1}{2} G_{\alpha \beta \gamma}{ }^{\pi \tau} G_{\pi \tau \rho}{ }^{\mu \nu}\right)$. In order to obtain a Riemannian massless equation for gravitational waves, we need the following condition to be fulfilled:

$$
\begin{aligned}
\delta g_{\alpha \beta ; \gamma \rho}= & -\delta g_{\nu \beta \mid \gamma} K_{\alpha \rho}^{\nu}-\delta g_{\alpha \nu \mid \gamma} K_{\beta \rho}^{\nu}-\delta g_{\alpha \beta \mid \nu} K_{\gamma \rho}^{\nu} \\
& -\left(\delta g_{\nu \beta} K_{\alpha \gamma}^{\nu}+\delta g_{\alpha \nu} K_{\beta \gamma}^{v}\right)_{; \rho} .
\end{aligned}
$$

Once the condition (26) is fulfilled, we obtain a massless gravitational wave equation in the absence of sources with respect to the Riemannian geometry:

$$
\emptyset g_{\alpha \beta}=0 .
$$


We must remark that Eq. (27) is effective on the Riemannian manifold and it is obtained from the distortion of an inner product over a given extended manifold. Equation (26) describes a very specific condition, which is not fulfilled in most cases, and therefore the induced effective gravitational wave equation on the Riemannian geometry might be massive in a more general case. It is important to notice that massive gravitons and the cosmological parameter are a possible alternative to explain the origin of dark energy (see [27]).

\section{Gravito-electrodynamics}

An interesting case, where the source of the geodesic equations (7), is given by electromagnetic fields $A^{\alpha}$. In this case the geodesic equations on the Riemannian manifold are

$\frac{\partial^{2} x^{\mu}}{\partial S^{2}}+\left\{\left\{_{\alpha \beta}^{\mu}\right\} U^{\alpha} U^{\beta}=q F^{\mu \beta} U_{\beta}\right.$,

where, in the absence of torsion $F_{\mu \beta}=A_{\beta \mid \mu}-A_{\mu \mid \beta}$ is the electromagnetic tensor. As we have seen before, this is the same equation as those without external force, but with the connections

$\Gamma_{\beta \gamma}^{\alpha}=\left\{\begin{array}{l}\alpha \\ \beta \gamma\end{array}\right\}-\frac{q}{2}\left(F_{\beta}^{\alpha} U_{\gamma}+F_{\gamma}^{\alpha} U_{\beta}\right)$,

in which $K_{\beta \gamma}^{\alpha}=-\frac{q}{2}\left(F_{\beta}^{\alpha} U_{\gamma}+F^{\alpha}{ }_{\gamma} U_{\beta}\right)$. We shall choose the simplest contortion in order to obtain a connection without torsion. In this case, the non-metricity is

$g_{\alpha \beta ; \gamma}=q F^{\nu}{ }_{\gamma}\left(U_{\alpha} g_{\nu \beta}+U_{\beta} g_{\nu \alpha}\right)$,

where the integrability condition of Eq. (37) is fulfilled ${ }^{1}$ :

$\oint \mathrm{d}|v|=\oint U \cdot V V^{\nu}[\mathrm{d}(A)]_{\nu \gamma} \mathrm{d} x^{\gamma}=0$,

1 We check the integrability for the connection of Eq. (29) by using Eq. (38), and taking into account the fact that $F=\mathrm{d}(A)+A \cdot T$. Then the condition of integrability must be checked by doing the following integration:

$$
\begin{aligned}
\oint_{\partial \Omega} \mathrm{d}|v| & =\oint_{\partial \Omega} U \cdot V V^{v}[\mathrm{~d}(A)]_{\nu \gamma} \mathrm{d} x^{\gamma} \\
& +\oint_{\partial \Omega} U \cdot V V^{\nu} A_{\mu} T_{\zeta \nu}^{\mu} \mathrm{d} x^{\zeta},
\end{aligned}
$$

where $\partial \Omega$ is a "loop", which is closed over the background geometry. This loop represents the closed path over which we parallel transport vector $V$, and $\Omega$ is the inner sub-manifold contained by $\partial \Omega$. In the present case the most simple option which guarantees integrability is the choice $T_{\zeta \nu}^{\mu}=0$. Then by the use of the Stokes theorem we obtain

$$
\begin{aligned}
\oint_{\partial \Omega} \mathrm{d}|v| & =\int_{\Omega} U \cdot V[\mathrm{~d}(V)]_{\kappa}^{\nu}[\mathrm{d}(A)]_{\nu \zeta} \mathrm{d} x^{\zeta} \wedge \mathrm{d} x^{\kappa}= \\
& =\int_{\Omega} U \cdot V\left(V^{\nu}{ }_{,} F_{\nu \zeta}-V^{\nu}{ }_{, \zeta} F_{\nu \kappa}\right) \mathrm{d} x^{\zeta} \otimes \mathrm{d} x^{\kappa}=0 .
\end{aligned}
$$

The first term in the r.h.s. of Eq. (31) as a result is found to be zero over a closed path. Then, by choosing $T_{\zeta v}^{\mu}=0$, we adopt the simplest choice that guarantees integrability. in which $F=\mathrm{d}(A)$, as usual in the absence of torsion.

Using Eq. (3), the action (1) takes the form

$\mathcal{S}=\frac{1}{2 \kappa} \int_{V} \mathrm{~d}^{4} x \sqrt{-g}(\overline{\mathcal{R}}+\Delta \mathcal{R})$.

Under charge conservation, we obtain

$$
\begin{aligned}
\Delta R= & \frac{1}{4} F^{\mu \nu} F_{\mu \nu} \\
& +\left(\left\{\begin{array}{l}
\mu \\
\rho \psi
\end{array}\right\} F_{\kappa \mu} U^{\rho}+\left\{\begin{array}{c}
\mu \\
\kappa \psi
\end{array}\right\} F_{\rho \mu} U^{\rho}-F_{\rho \kappa, \psi} U^{\rho}\right) g^{\kappa \psi} .
\end{aligned}
$$

The first term in (34) is interpreted as an effective electromagnetic Lagrangian of matter for the effective Riemannian dynamics: $\overline{\mathcal{L}}=\frac{1}{4} F^{\mu \nu} F_{\mu \nu}$. The second one leads to the $\Lambda_{2}(x)$ contribution in the cosmological parameter. An observer which moves on a Riemannian manifold could not explain the origin of the two terms. The geometrical action originates with the connection of Eq. (29) describing a scenario which induces the effective dynamics observed by a Riemannian observer over a background metric with external electromagnetic fields and nonzero cosmological parameter:

$\delta \mathcal{S}=\int \mathrm{d}^{4} x \sqrt{-g}\left(\bar{G}_{\mu \nu}+\Lambda_{1}(x) g_{\mu \nu}+k \bar{T}_{\mu \nu}\right) \delta g^{\mu \nu}$.

Here, $k \bar{T}_{\mu \nu}=-2 \frac{\delta \overline{\mathcal{L}}}{\delta g^{\mu \nu}}+g_{\mu \nu} \overline{\mathcal{L}}$ is an effective energymomentum tensor for electromagnetic fields. On the Riemannian manifold the electromagnetic dynamics is given by the equations:

$$
\begin{gathered}
d(* F)=* J, \\
d(F)=0 .
\end{gathered}
$$

Notice that current terms originate with the non-Riemannian part of the connections (9). This is an expected behavior because such an extra part is related to electrodynamics by the definition in Eq. (29). The second line of these equations tells us that magnetic monopoles cannot be present, due to the absence of torsion on the Riemann manifold.

\section{Final comments}

We have considered the variation of the geometrical contribution of the $\mathrm{EH}$ action, taking into account the conditions of integrability in the boundary conditions. It is interesting that some physical phenomena can be described when we consider a displacement from a background Riemann manifold, such that the non-metricity on the extended manifold is nonzero. In particular, in this work we have dealt with emergent gravitational waves and electromagnetic interactions in a classical description. In the second case the absence of monopoles is compatible with the scenario studied by Ponce 
de León in [28]. Of course, we have not made any assumption as regards the metric tensor. Our results remain valid for both a spherically symmetric and a static metric, which enables us to use the Ponce de León results in a charged perfect fluid interpretation. Therefore, in a co-moving frame we perceive a central massive and charged object, compatible with an electric monopole surrounded by a perfect fluid that can be obtained from the effective equation (36). In the case in which our metric were not spherically symmetric and static, we would describe the electromagnetic interaction from a geometrical source in order to obtain effective gravitational waves, according to Eq. (25). In the case of gravitational waves the action of Eq. (1) is expressed over a non-Riemannian geometry, implying that the equivalent action over a Riemannian geometry must have extra terms. This is equivalent to the one exposed by Visser in [29]. In our case such terms are induced from the extended geometry, which originates with the non-metricity of the extended manifold (i.e., the covariant derivative of the tensor metric on the extended manifold is nonzero: $g_{\alpha \beta ; \gamma} \neq 0$ ). This means that the physical interpretation of phenomena is determined by the geometrical description of reality in which the observer describes the physical system. This interpretation of the physical phenomena could be very interesting in a cosmological framework, For instance, the existence of a cosmological parameter $\Lambda(x)$ in the universe could be interpreted as an empirical proof of a non-conservative norm of vectors and tensors (originating with a nonzero non-metricity), along with the expansion of the universe without physical sources, described on a Riemannian manifold, in agreement with Eq. (21). Another possible interpretation is a universe with cosmological parameter $\Lambda_{1}(x)$, such that the expansion is produced by a physical source $\bar{T}_{\alpha \beta}$, in agreement with the Eq. (17), explained on a Riemann manifold. However, this topic is beyond the scope of this work, and it deserves a more rigorous study in future work.

Acknowledgements The authors acknowledge CONICET, Argentina (PIP 11220150100072CO) and UNMdP (EXA852/18) for financial support.

Data Availability Statement This manuscript has no associated data or the data will not be deposited. [Authors' comment: We have no used any particular data to make this article.]

Open Access This article is distributed under the terms of the Creative Commons Attribution 4.0 International License (http://creativecomm ons.org/licenses/by/4.0/), which permits unrestricted use, distribution, and reproduction in any medium, provided you give appropriate credit to the original author(s) and the source, provide a link to the Creative Commons license, and indicate if changes were made.

Funded by SCOAP ${ }^{3}$.
Appendix A: Conditions of integrability in boundary terms

The condition of integrability expresses the fact that we can univocally assign a norm to any vector in a given point, which can be written as

$\oint \mathrm{d}|V|=0$.

Non-integrability is due to the fact that, when we parallel transport of a vector field along a closed path, the "norm" could change in proportion to the non-metricity according to

$$
\begin{aligned}
\oint \mathrm{d}|V| & =\frac{1}{2|V|} \oint g_{\nu \pi ; \rho} V^{\nu} V^{\pi} \mathrm{d} x^{\rho} \\
& =\frac{1}{|V|} \oint f \cdot V U \cdot V U_{\rho} \mathrm{d} x^{\rho} .
\end{aligned}
$$

It is easy to see that orthogonality between $f$ and $V$, or between $U$ and $V$, grants the nullity of the integral in Eq. (38), but $V$ must be any vector field, and therefore we cannot say that $f \cdot V=0$ or $U \cdot V=0$. We could think that Eq. (38) is providing us with a sufficient condition over the possible velocity of observers (the field of the four-velocity $U$ could be non-rotational), in order to obtain integrability. However, it is not necessary, as we shall see later, when we analyze gravitomagnetic interactions. A non-intuitive fact is that torsion plays a significant role in integrability. We are performing a closed integral in order to show how the norm of a vector changes, but a closed path must be affected by torsion. We could expect that a Burgers vector arises. This is a measure of how a given path, which is closed in a torsion-free geometry, fails to be closed in the torsional one. Then an extra term appears in Eq. (38), and such a term could complete the integral in order to be really closed. We say that torsion does not affect the geodesics, but it affects integrability, so that the choice of an appropriate torsion could be an ingredient which as a result is found to be appropriate to make the theory integrable in the case that it is possible to obtain a torsion that compensates the integral of the r.h.s. of Eq. (38). In the example developed in Sect. (5), we show that the most simple choice for a given connection (i.e., the null torsion choice), is a good choice (but not the unique choice), in which integrability is maintained [see footnote 1].

\section{References}

1. J.A. Wheeler, Ann. Phys. 2, 604 (1957)

2. J.A. Wheeler, Superspace and the Nature of Quantum Geometrodynamics, in Batelle Rencontres, 1967, in: Lect, ed. by C.M. De Witt, J.A. Wheeler (Math. Phys. W. A. Bemjamin, New York, 1968) 
3. G.W. Gibbons, Lect. Notes Phys. 160, 145 (1968)

4. I. Rácz, Class. Quantum Gravity 32, 015006 (2015)

5. E. Prugovečki, Ann. Phys. 110, 102 (1978)

6. E. Prugovečki, Phys. Rev. D 18, 3655 (1978)

7. E. Prugovečki, Nuo. Cim. A97, 597 (1987)

8. A. Wheeler, Ann. Phys. 2, 604 (1957)

9. C. Rovelli, Living Rev. Relat. 1, 1 (1998)

10. C. Rovelli, L. Smolin, Phys. Rev. D 52, 5743 (1995)

11. L.S. Ridao, M.R.A. Arcodía, J.M. Romero, M. Bellini, Eur. Phys. J. Plus 133, 507 (2018)

12. J.W. York, Phys. Rev. Lett. 16, 1082 (1972)

13. G.W. Gibbons, S.W. Hawking, Phys. Rev. D 10, 2752 (1977)

14. M. Israelit, Found. Phys. 35, 1769 (2005)

15. J.M. Romero, M. Bellini, Eur. Phys. J. C 74, 3043 (2014)

16. J.M. Romero, M. Bellini, Phys. Dark Univ. 15, 47 (2017)

17. L.S. Ridao, M. Bellini, Phys. Lett. B 751, 565 (2015)

18. L.S. Ridao, M. Bellini, Astrophys. Space Sci. 357, 94 (2015)

19. A. Einstein, Sitzungsber. Preuss. Akad. Wiss. Berlin (Math.Phys.) 1918: 154 (1918)
20. A. Einstein, N. Rosen, J. Franklin Inst. 223, 43 (1937)

21. B.P. Abbott et al., (Virgo, LIGO Scientific). Phys. Rev. Lett. 116, 061102 (2016)

22. B.P. Abbott et al., (Virgo, LIGO Scientific). Phys. Rev. Lett. 116, 241103 (2016)

23. B.P. Abbott et al., (Virgo, LIGO Scientific). Phys. Rev. X 6, 041015 (2016)

24. B.P. Abbott et al., (VIRGO, LIGO Scientific). Phys. Rev. Lett. 118, 221101 (2017)

25. B.P. Abbott et al., (Virgo, LIGO Scientific). Phys. Rev. Lett. 119, 141101 (2017)

26. B.P. Abbott et al., (Virgo, LIGO Scientific). Phys. Rev. Lett. 119, 161101 (2017)

27. M.E.S. Alves, O.D. Miranda, J.C.N. de Araujo, Phys. Lett. B 700, 283 (2011)

28. J. Ponce de León, Phys. Rev. D 95, 124015 (2017)

29. M. Visser, Class. Quantum Gravity 30, 1717 (1998) 